\title{
openheart Significant reduction in hospital- acquired thrombosis: impact of national risk assessment and real-time feedback
}

\author{
Huw Raymond Rowswell, Timothy J C Nokes
}

\begin{abstract}
- Additional material is published online only. To view please visit the journal online (http://dx.doi.org/10.1136/ openhrt-2017-000653)
\end{abstract}

To cite: Rowswell HR, Nokes TJC. Significant reduction in hospital-acquired thrombosis: impact of national risk assessment and realtime feedback. Open Heart 2017;4:e000653. doi:10.1136/ openhrt-2017-000653

Received 5 May 2017 Revised 1 September 2017 Accepted 12 September 2017

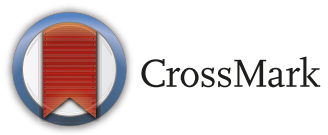

Department of Haematology, Plymouth Hospitals NHS Trust, Plymouth, UK

Correspondence to Mr Huw Raymond Rowswell; huw.rowswell@nhs.net

\begin{abstract}
Objective Since 2010, National Health Service hospitals in England have been incentivised to risk assess for Venous Thromboembolism (VTE) in all adult patients admitted, using a national tool. We studied the impact of this, together with local real-time reporting of VTE events to senior clinicians, on cases of hospital-acquired thrombosis (HAT) diagnosed, since 2010.

Methods This was an observational cohort study reviewing all cases of VTE diagnosed between January 2010 and December 2016 in a single teaching hospital. These were matched against the number of patients admitted to produce crude incidence rates per thousand admissions. Similarly, all cases associated with inadequate thromboprophylaxis (TP) measures were documented over the same period.

Results By the end of 2010, with 70\% compliance with VTE risk assessment, improving to $90 \%$ (the national target) early in 2011, there were 217 HAT events from 103845 admissions. In 2016, there were 176 HAT events from 119128 admissions, being a significant reduction, relative risk (RR) $0.718(95 \% \mathrm{Cl} 0.589$ to $0.875 ; \mathrm{p}=0.001)$. In 2010, there were 50 of 217 HAT events associated with inadequate TP, falling to 7 of 176 in 2016, also a significant reduction, RR $0.140(95 \% \mathrm{Cl} 0.065$ to 0.300 ; $\mathrm{p}=0.0001$ ).

Conclusions National guidance on VTE prevention and mandatory risk assessment linked to local real-time reporting of VTE events are associated with significant reductions both in total HAT events and those associated with inadequate TP.
\end{abstract}

\section{INTRODUCTION}

In 2010, the National Institute for Health and Care Excellence (NICE) published guidance on the prevention of Venous Thromboembolism (VTE) in hospitalised patients. ${ }^{1}$ This was supported by a financial incentive, from the Commissioning for Quality and Innovation (CQUIN),${ }^{2}$ to achieve $90 \%$, later increased to $95 \%$, compliance with documented VTE risk assessment using a national tool. The risk assessment tool mandated by $\mathrm{NICE}^{3}$ covers all adult patients admitted to hospital with separate guidance for maternity patients from the Royal College of Obstetricians and Gynaecologists. ${ }^{4}$ The risk assessment tools initially cover patient mobility and whether

\section{KEY MESSAGES}

What is already known about this subject?

- Hospitalised patients are at an increased Venous Thromboembolism (VTE) risk, and the use of appropriate prophylaxis, chemical and/or mechanical, can reduce this. Since mandatory risk assessment was introduced in England, studies have suggested that this has decreased both number of hospital-acquired thromboses (HATs) and 90 -day mortality from VTE.

What does this study add?

- Seven years after mandatory VTE risk assessment was introduced, these data from a large teaching hospital have identified a significant reduction in HAT. Over the last 2 years, this has subsequently plateaued as one might expect, acknowledging that not all these events can be prevented. We believe that the novel use of real-time feedback to clinicians regarding HAT occurring in their patients has been partly instrumental in reducing these events. Moreover, there has also been a significant reduction in HAT events associated with inadequate thromboprophylaxis (TP). To our knowledge, such significant VTE metrics have not been published from other institutions.

How might this impact on clinical practice?

- The use of real-time feedback for thrombotic events (HAT) to the discharging clinician is a relatively simple and cost-effective process to reduce both HAT events and particularly those associated with inadequate TP. With the introduction of duty of candour within hospitals in England, a reduction in avoidable harm is a positive step.

this has changed relative to normal state. This is followed by specific risk factors for thrombosis, both patient related such as age $>60$ years, personal or family history of VTE and presence of a significant medical co-morbidity. Then, admission-related risks are assessed including surgical time and ongoing reduction in mobility. Finally, the bleeding risk is also assessed: both patient related such as active bleeding or concurrent use of 
anticoagulation and admission related such as neurosurgery and spinal surgery admissions or lumbar puncture procedure. Once the risk assessment has been completed, a decision is made about thromboprophylaxis (TP) application, dependent on risk of bleeding and admission type whether to use chemical only, a combination of both chemical and mechanical or mechanical only. Guidance from NICE is also used as this covers different admitting specialties concerning what prophylaxis to use as well as any patient safety concerns. A clinical nurse specialist was appointed to drive the VTE prevention programme, with specific roles in producing real-time data on hospital-acquired thrombosis (HAT), determine incidence rates, both total HAT, VTE by admitting specialty and provide feedback to clinicians about these VTE events. HAT is defined as: VTE events diagnosed within hospital, not present on admission, or within 90 days of hospital discharge. ${ }^{5}$ Several studies have suggested that a reduction in HAT events is related to the introduction of the mandatory risk assessment. ${ }^{67}$ We report on 7 years of HAT data following the introduction of both VTE risk assessment and real-time feedback to clinicians of such events.

\section{SUBJECTS AND METHODS}

This was an observational cohort study reviewing all VTE cases diagnosed within Plymouth Hospitals National Health Service (NHS Trust), a large teaching hospital with 900 beds. As this was a quality improvement project and only routinely collected clinical data were used, it was not necessary to seek ethics approval, though the audit was registered with the hospital audit department. Positive VTE cases are generated from daily, retrospective review of the preceding day's targeted radiography records on the hospital radiology system, 'CRIS'. CT pulmonary angiograms (CTPAs), Doppler ultrasounds, both lower and upper limbs and ventilation perfusion scans are reviewed to identify VTE events. In addition, incidental VTE findings, from CT scans of the thorax abdomen and pelvis, predominantly for cancer screening, were also included. All such radiography examinations were included from 1 January 2010 to 31 December 2016. In addition, the total number of scans, both positive and negative, is recorded daily to look at trends in the number performed annually and the percentage of positive findings. The hospital policy for suspected Pulmonary Embolism (PE) was updated in 2011, so patients with a normal chest X-ray, no significant cardiorespiratory disease and a high or intermediate pretest probability and positive d-dimer would have a V/Q scan rather than a CTPA. Positive VTE events are then crosschecked against the hospital patient identification system to identify whether HAT criteria are met.

The risk assessment tool appears on the second page of the adult inpatient drug chart (see online supplementary file 1 for copy), and the low-molecular-weight heparin (LMWH) used for chemical VTE prophylaxis is preprinted as the first drug on the chart with the instruction to cross this drug off if not indicated. However, as there is no dose, duration or frequency printed, this would not be routinely administered. The drug chart was amended to include VTE risk assessment and guidance on chemical prophylaxis prescription in the summer of 2010 in light of the new guidance on this from NICE in the same year. This followed extensive small-scale testing initially among the medical admissions unit where the largest number of medical patients is admitted to the hospital. The clinical nurse specialist worked closely in this area for several months together with the junior doctors and implementing feedback and amendments to the chart until it was rolled out hospital-wide by the end of 2010 . Data collection on compliance with a documented VTE risk assessment happens in three ways. The majority of data are obtained from a mandatory field within the electronic discharge system that record whether a VTE risk assessment on admission took place. There is also a large number of low-risk, day case patients who do not have a general anaesthetic and covered under a generic low-risk assessment agreed with the hospital medical director. Finally, the official hospital coding department collect some data by checking that the drug chart has been completed. Regular spot-check audit is carried out to ensure that compliance with VTE risk assessment has been made.

The hospital already had protocols and policies in place regarding VTE prophylaxis, prior to NICE guidance. This required all medical patients aged over 40, admitted to hospital, be prescribed VTE prophylaxis, generally LMWH. In addition, there were separate policies for surgical patients, usually involving antiembolism stockings as well as chemical prophylaxis in the form of LMWH.

The hospital serves a direct population of $475000 .^{8}$ The number of patients admitted annually is used to calculate crude incidence rate (CIR) (per 1000 patient admissions) when matched with annual HAT numbers. The patient admission numbers and HAT events are for adult patients aged 18 and over, being the population group that NICE guidance on risk assessment relates. Since June 2010, there has been mandatory data collection by NHS England using an online system called Unify $2 .{ }^{9}$ For all HAT events, a basic root cause analysis (RCA), using another standardised tool, is carried out. This determines whether a VTE risk assessment took place, what prophylaxis was used, if that was appropriate and what VTE risk factors were present. Feedback from all HAT cases is then provided to the discharging clinician using standard email format. Errors or omissions when identified require the discharging clinician to carry out a more thorough RCA, reviewing whether VTE prevention was appropriate and giving feedback to the VTE prevention team and patient, as appropriate. Inadequate VTE prevention is either where the prescription for VTE prevention has not been completed or the dose or type administered not appropriate for the patient. There is an increased prophylactic dose for patients weighing 
over $100 \mathrm{~kg}$ dependent on their renal function. Missed prescribed doses of TP obviously provide another source of inadequate TP. Clearly, cases associated with a high bleeding risk procedure would not be included. All HAT events are reviewed by the VTE prevention team-usually by the CNS and hospital VTE lead, a consultant haematologist. Annually, all HAT cases by admitting specialty are compared against the number of patients admitted, to ascertain the risk of HAT, focusing on those areas with the highest rates, requiring specific assistance from the VTE prevention team. Those areas identified as having the highest percentage HAT events relative to patients admitted were reviewed carefully for concerns related to risk assessment, reassessment or prophylaxis

\section{Case study}

Following 12 months of data collection on HAT by specialty, we identified neurosurgery as having the highest CIR. We worked with a link nurse, a register and neurosurgery consultant to improve reassessment of VTE risk within these patients. The link nurse and CNS also took part in consultant ward rounds at times to highlight the importance of reassessment and appropriate TP prescription. The CIR improved significantly over the following years.

\section{STATISTICAL ANALYSIS}

Outcome data were collected in a spreadsheet, cleaned and then exported into SPSS V.21 for statistical analysis. CIRs were calculated with $95 \%$ CIs, and then $\chi^{2}$ test was used to compare the data from the beginning of the study in 2010 with data from 2016, looking for significant differences and calculate ORs and RRs.

\section{RESULTS}

A national return on the number of patients having a VTE risk assessment was mandated for all hospitals in England from June 2010, and this continues presently. ${ }^{9}$ The first month of data from Plymouth showed 5\% of patients being risk assessed, improving to $70 \%$ by the end of 2010 and rising to above $90 \%$ by February 2011. Subsequently, risk assessment improved to greater that $95 \%$ when the target was changed in 2014. Despite the

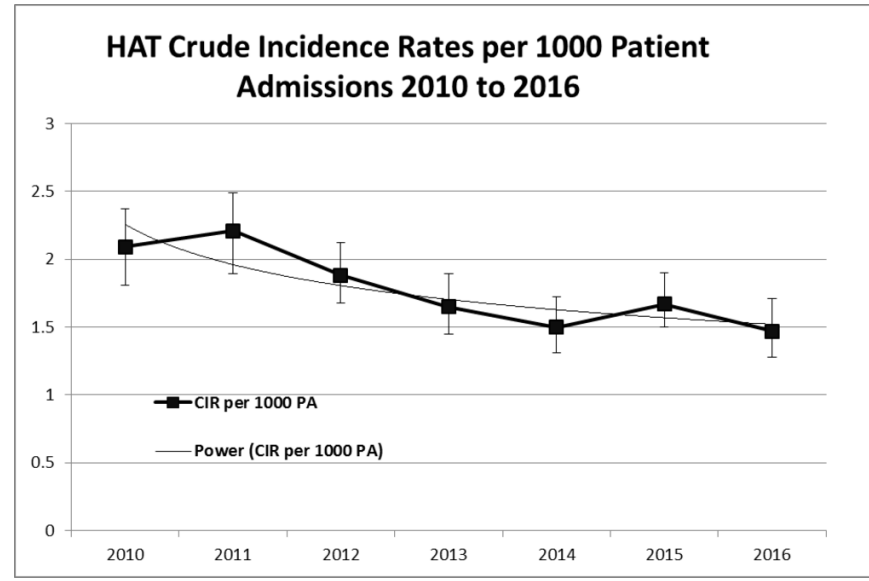

Figure 1 CIRs for HAT 2010-2016. CIR, crude incidence rate; HAT, hospital-acquired thrombosis.

initial poor adherence to VTE risk assessment, snapshot audits of 300-400 patients monthly showed that rates of appropriate TP were between $94 \%$ and $98 \%$ from patients across all specialties. The updated NICE guidance in 2010 stated that a formal risk assessment was now required, with guidance for medical patients with no change to normal mobility, no longer requiring TP. Also, with risk assessment and TP prescription being required on admission, there was an increase in use of TP particularly in shorter-stay patients.

Table 1 shows the total adult patient admissions and HAT rates for 2010-2016, together with CIR for HAT events over that time period. There were 902 PE diagnoses, and 472 deep vein thrombosis (DVT) diagnoses of which 20 were upper arm thrombosis. Of the 50 patients with HAT associated with inadequate TP in 2010, 26 were associated with at least one omitted, prescribed dose of chemical prophylaxis. There were 24 cases where either no prophylaxis was prescribed or an incorrect/inadequate dose was prescribed. In 2016, for the seven cases of inadequate TP, two involved missed doses and five where an inadequate dose was prescribed, mainly due to low doses of LMWH, prescribed for obese patients.

This information is shown in graphical form in figure 1.

The OR for increased risk of HAT in 2010 compared with 2016 was calculated as 1.41 (95\% CI 1.16 to 1.73$)$,

\begin{tabular}{lclllll}
\hline Table $\mathbf{1}$ & CIRs for HAT in 2010-2016 & & & & \\
\hline Year & Total Pt $(\mathbf{n})$ & Day cases, $\mathbf{n}(\%)$ of PA & Total HAT (n) & CIR per 1000 PA & $\mathbf{9 5 \% ~ C I ~}$ & ITP(n) \\
\hline 2010 & 103845 & $38839(37)$ & 217 & 2.09 & 1.81 to 2.37 & 50 \\
2011 & 108428 & $42127(39)$ & 240 & 2.21 & 1.89 to 2.49 & 48 \\
\hline 2012 & 114959 & $45674(40)$ & 216 & 1.88 & 1.68 to 2.13 & 24 \\
\hline 2013 & 103887 & $40946(39)$ & 171 & 1.65 & 1.45 to 1.89 & 10 \\
2014 & 114080 & $43967(39)$ & 171 & 1.5 & 1.31 to 1.72 & 14 \\
2015 & 114112 & $46832(41)$ & 190 & 1.7 & 1.50 to 1.90 & 8 \\
2016 & 119128 & $46685(39)$ & 169 & 1.42 & 1.22 to 1.65 & 7 \\
\hline
\end{tabular}

CIR, crude incidence rate; HAT, hospital-acquired thrombosis; ITP, inadequate thromboprophylaxis; PA, patient admission; Pt, patient. 
and $\chi^{2}$ test with Yates correction for this showed $\chi^{2}$ $(\mathrm{n}=222973)=11.47, \quad(\mathrm{p} \leq 0.001)$. This demonstrates a significant reduction in CIR. For inappropriate TP, the OR was 7.23 (95\% CI 3.19 to 16.401 ), and $\chi^{2}$ test with Yates correction was $\chi^{2}(\mathrm{n}=393, \mathrm{p} \leq 0.0001)$, again showing a significant reduction. Over the 7 years, this gives a significant $R R$ reduction of 0.293 and a significant absolute risk reduction of $0.00061(\mathrm{p}<0.01)$ of developing HAT per admission in 2010 compared with 2017.

The percentage of positive scans for VTE from the targeted VTE investigations remained relatively similar over the 7 years with no trends: $\mathrm{V} / \mathrm{Q}$ scans ranging from $18 \%$ to $23 \%$ positive events, CTPA from $16 \%$ to $21 \%$ and Doppler ultrasounds (DUS) from 9\% to 13\%, However, looking at number of scans carried out annually, there has been a $62 \%$ increase in CTPA examinations, from 1235 to 2005, and a 33\% increase in V/Q tests, from 723 to 965 , between 2010 and 2016. Over the same period, DUS have only seen a $5 \%$ increase in numbers performed from 3257 to 3433 . Over the same period, the total number of VTE events has increased from 703 in 2010 to 862 in 2016, with total PE increasing from 381 to 468 (22\%) and total DVT from 322 to 384 (19\%).

\section{Case study results}

HAT rates in neurosurgery for 2010 were 19 events from 2343 (CIR $=0.81 \%)$, and snapshot audit of the area over the year showed poor compliance with risk assessment: only 20\% VTE risk assessments were completed, and appropriate TP was $60 \%-70 \%$, assessing thrombosis and bleed risk. Following work by the CNS, ward link nurse and Trust VTE lead around the importance of reassessment as the bleed risk reduces postadmission, HAT rates associated with this specialty reduced over the following years. In 2011, there were 16 events from 2098 admissions $(0.76 \%)$ and in 201212 events from 2250 admissions $(0.53 \%)$. The most recent figures from 2016 show 10 events from 2315 admissions $(0.43 \%)$.

\section{DISCUSSION}

In this observational case control study, we demonstrated a significant reduction in HAT events, expressed as a percentage of total patient numbers (CIR). More importantly, those events associated with inadequate TP over the 7 years studied have also significantly reduced. The reduction in HAT events appears to be reaching a plateau in 2015-2016. The likely explanation for this is that, for at-risk inpatients, TP can reduce VTE by about $65 \%,{ }^{10}$ so not all VTE events can be prevented. This is despite an increase in targeted VTE scans and more overall VTE events being diagnosed.

The hospital radiology system was used to collect VTE data. Coding was considered to identify positive VTE events, but this is less accurate as it frequently does not differentiate between old and new thromboses. ${ }^{8}$ Radiology is a more accurate methodology for producing VTE outcome metrics ${ }^{9}$ and can deliver results in real time.
Prior to 2010, the hospital already had protocols in place around the prescribing of VTE prophylaxis. This 'opt out' policy may explain the initial poor compliance with risk assessment but good results for appropriate TP.

In the first year of collecting data on VTE metrics, 50 cases (23\% of all HAT) were associated with either a missed dose of anticoagulant or inappropriate TP prescription as defined in the Subjects and methods section. Missed doses are those detailed in the Subjects and methods section, though during a long inpatient stay, the omission of a single dose of TP is unlikely to be the cause of the thrombosis, but an association needs documenting. However, a paper by Louis et $a l^{11}$ describes missed doses as being common and a strong independent risk factor for developing DVT-the only identified risk factor that can be reduced by the admitting team.

Feedback to clinicians has appeared to have improved awareness of VTE, as the thrombosis is frequently managed by a different team and the discharging clinician not aware of the event. For those events with errors, the team is required to complete a RCA to review the individual cases.

We have also worked with specialties with the highest HAT rates over the duration of this patient safety initiative. The first is detailed in the case study around neurosurgery, but similarly, in colorectal surgery, there had been a number of VTE events associated with patients having active cancer. Working with the team to ensure extended prophylaxis (for 4 weeks postdischarge) for these patients resulted in a reduction in such events.

Over the study period following publication of NICE guidance and adoption by the hospital, there has been an increase in VTE investigations (particularly for PE) associated with an increase in PE diagnoses. Over the same period, there has only been a small increase in scans for suspected DVT. This may reflect a greater awareness of VTE risk associated with hospital admissions, and PEs being potentially life threatening, it is not possible to calculate how many scans were for potential HAT events, only total radiological investigations. The pick-up rates over the study period have remained similar, however, suggesting more investigations and more total VTE events. Similarly, community-acquired VTE has increased with an increase in the clinical suspicion for VTE.

The introduction of 'duty of candour' to hospitals in England happened in November $2014,{ }^{12}$ which reinforces a legal duty to be open and honest to patients and families following episodes of patient harm. This means that, for HAT events associated with errors or omissions, the patient needs to be involved in the investigation process and an apology given if potential mistakes are proven. The reduction in the number of HAT events associated with adequate prevention from 50 in 2010 to 7 in 2016 reduces the need for duty of candour to be applied for HAT. This is an important patient safety issue and may lead to a reduction in litigation associated with HAT events where errors were identified. 
In a paper by Lester et $a l^{7}$ there was a significant reduction in death from VTE within 90 days of hospital discharge in patients admitted for at least 3 days. This NHS hospital met the quality standard at the time of at least $90 \%$ documented VTE risk assessment, put forward as a likely cause. Another paper by Roberts et $a l^{6}$ reviewed HAT events in a large teaching hospital, demonstrating that when risk assessment improved from $40 \%$ compliance to greater than $90 \%$ and sustained at this level, this led to a significant reduction in HAT events. They also showed that the number of such HAT events associated with inadequate prophylaxis fell significantly from $37.5 \%$ to $22.4 \%$. A further study on hospital-acquired VTE, Heit et al (2013) ${ }^{13}$, using incidence-based modelling, reported 600000 VTE events in the USA with more than two-thirds, 419825, being hospital associated. A French study over 2 years ${ }^{14}$ covering some $77 \%$ of the population showed a VTE incidence rate of 1.84 cases per 1000 subjects but no data on how many were hospital acquired. A study looking at the effect of immobility and VTE by Engbers et $a l^{15}$ described a HAT rate of $31.4 \%$ and followed up patients for 3 months postdischarge. Interestingly, they described that the highest rate of thrombosis was seen between 1 and 3 months postdischarge unlike the findings by Sweetland et al, ${ }^{16}$ who describe highest rates in the first 6 weeks postdischarge. Finally, the RIETE registry by Maestre $e t a l^{17}$ compared inpatient with outpatient VTE and showed $29 \%$ of thrombotic events associated with hospital admission and that these patients had a higher overall mortality. However, while it follows up patients for 3 months from diagnosis looking at mortality, it does not collect any data on VTE events that are diagnosed postdischarge and is thus likely to under-represent the problem. Looking at information from Medicare, there are significant differences in how the USA defines whether treatment is on an inpatient or outpatient basis. ${ }^{18}$ For example, many overnight stays including a critical care admission for monitoring would still be considered as outpatients, which would differ from the definition we use in England. In a study looking at number of VTE events in the USA between 2005 and 2010, Heit et al ${ }^{19}$ showed hospital admission associated with $50 \%$ of VTE and nearly $75 \%$ of events diagnosed postdischarge, stressing the importance of collecting data postdischarge. This paper highlighted that, despite near universal compliance on use of TP in medical inpatients, there was no significant change in the number of VTE events, which is at variance with our findings. They do suggest that better identification of patients at highest risk is needed to use extended prophylaxis on the population that would benefit most from this, similar to our high-risk surgical patients previously identified.

For future study, it would be useful to break down HAT annually by specialty to see if the reductions in events are associated with particular admitting types or whether the reduction is seen across the board.

Surveillance bias may impact on reporting and investigation of VTE events, with a suggestion that, if TP rates are high, there will be a higher threshold for investigating possible thrombosis and vice versa. ${ }^{20}$ Within the hospital following adoption of NICE guidance in 2010 on risk assessment and appropriate prophylaxis, this did lead to some changes to TP prescription. As the majority of inpatients were already receiving VTE prophylaxis, this was not seen as a significant change. The major difference was in medical inpatients that had no significant change to mobility no longer requiring treatment. Local audit has not revealed any major changes to the percentage of patients receiving TP, but this must be acknowledged as a potential limitation.

We acknowledge as an observational study that there will be some HAT events diagnosed by other healthcare providers and therefore not known to us or included within the data. Our hospital covers a large geographical area so the majority of VTE events diagnosed within 90 days of discharge, to meet HAT criteria, present to our hospital. We do receive feedback from our nearest hospital about any VTE events presenting there. We acknowledge that some data may be missing, but this is likely to be similar each year and is thus unlikely to impact greatly on the results shown. This is particularly a concern in this tertiary referral centre.

\section{CONCLUSIONS}

Over the 7 years studied, the VTE metrics from Plymouth Hospitals demonstrate significant reductions in the CIR for HAT events. More importantly, a significant reduction in HAT events associated with inadequate TP has been documented. With the introduction of duty of candour to NHS, all such events require reporting and disclosure to patients, so there is an increased imperative to prevent them. The combination of a national VTE risk assessment tool since $2010^{3}$ and the CQUIN imitative ${ }^{2}$ has contributed to the reduction in HAT. However, we believe that the production of real-time outcome data and clinician feedback has provided the most important contribution toward this significant improvement in patient safety.

In view of the plateau in HAT events, further study could look in more detail at each HAT event to see whether any common themes or specific risk factors are identified. We may identify a cohort of patients who would benefit from extended prophylactic TP postdischarge, in the same way as that for cancer-associated surgery. Looking at HAT events by admitting specialty may identify patients who would benefit from extended VTE preventative measures.

Contributors HRR collected the data and calculated the statistics; TJCN checked the statistics; HRR and TJCN wrote and reviewed the paper.

Competing interests None declared.

Provenance and peer review Not commissioned; externally peer reviewed.

Data sharing statement All data are provided within the results section of the paper in full covering total annual admissions, total number of hospital associated thrombosis and total events associated with inappropriate care. Other documents that are available are the root cause analysis template and the standard letters sent to clinicians regarding HAT events. These documents are available immediately after publication by email request to the corresponding author. 
Open Access This is an Open Access article distributed in accordance with the Creative Commons Attribution Non Commercial (CC BY-NC 4.0) license, which permits others to distribute, remix, adapt, build upon this work non-commercially, and license their derivative works on different terms, provided the original work is properly cited and the use is non-commercial. See: http://creativecommons.org/ licenses/by-nc/4.0/

(c) Article author(s) (or their employer(s) unless otherwise stated in the text of the article) 2017. All rights reserved. No commercial use is permitted unless otherwise expressly granted.

\section{REFERENCES}

1. National Institute for Health and Care Excellence. Venous thromboembolism: reducing the risk: reducing the risk of venous thromboembolism (deep vein thrombosis and pulmonary embolism) in patients admitted to hospital | guidance and guidelines | NICE. http://www.nice.org.uk/guidance/CG92\#.VAiWbOYrwGY.mendeley (accessed 4 Sep 2014).

2. Department of Health. Using the Commissioning for Quality and Innovation (CQUIN) payment framework-Guidance on national goals for 2011/12. Introd. to Natl. CQUIN Goals. 2010 http:// webarchive.nationalarchives.gov.uk/20130107105354/http:/www. dh.gov.uk/prod_consum_dh/groups/dh_digitalassets/@dh/@en/@ps/ documents/digitalasset/dh_123009.pdf (accessed 1 Aug 2014).

3. Department of Health. Risk assessment for venous thromboembolism in hospitalised patients. risk assemment venous thromboembolism hosp.patients. 2010 http://reception.e-Ifh.org. uk/vte/content/VTE 03 01/d/ELFH Session/457/tab 549.html (accessed 15 Jun 2017).

4. Reducing the risk of venous thromboembolism during pregnancy and the puerperium green-top guideline No. 37a. 2015 http:// reception.e-Ifh.org.uk/vte/content/VTE_03_01/d/ELFH_Session/457/ tab_549.html (accessed 15 Jun 2017).

5. Arya R, Hunt B. Venous thromboembolism prevention a guide to deliverying the CQUIN goal. $2010 \mathrm{http}: / /$ www.vteexemplarcentres. org.uk/manual_uploads/A guide to delivering the CQUIN goal.pdf (accessed 3 Apr 2015).

6. Roberts LN, Porter G, Barker RD, et al. Comprehensive VTE prevention program incorporating mandatory risk assessment reduces the incidence of hospital-associated thrombosis. Chest 2013;144:1276-81.

7. Lester W, Freemantle N, Begaj I, et al. Fatal venous thromboembolism associated with hospital admission: a cohort study to assess the impact of a national risk assessment target. Heart 2013;99:1734-9.

8 Plymouth Hospital NHS. Plymouth Hospitals NHS Trust. 2014.http:// www.plymouthhospitals.nhs.uk/ourorganisation/Pages/Home.aspx (accessed 20 Nov 2014).

9. NHS England. Statistics Venous Thromboembolism (VTE) risk assessment. https://www.england.nhs.uk/statistics/statistical-workareas/vte/ (accessed 16 Aug 2017).

10. Guyatt $\mathrm{GH}, \mathrm{Akl} \mathrm{EA}$, Crowther $\mathrm{M}$, et al. . Executive summary: antithrombotic therapy and prevention of thrombosis, 9th ed: American college of chest physicians evidence-based clinical practice guidelines. Chest 2012;141:7S-47.

11. Louis SG, Sato M, Geraci T, et al. Correlation of missed doses of enoxaparin with increased incidence of deep vein thrombosis in trauma and general surgery patients. JAMA Surg 2014;149:365.

12. Department of Health. Introducing the statutory duty of candour. March 2014 https://www.gov.uk/government/uploads/system/ uploads/attachment_data/file/295773/Duty_of_Candour_ Consultation.pdf.

13 Heit JA. Venous thromboembolism: disease burden, outcomes and risk factors. J Thromb Haemost 2005:3:1611-7.

14 Bouée S, Emery C, Samson A, et al. Incidence of venous thromboembolism in France: a retrospective analysis of a national insurance claims database. Thromb J 2016;14.

15 Engbers MJ, Blom JW, Cushman M, et al. The contribution of immobility risk factors to the incidence of venous thrombosis in an older population. J Thromb Haemost 2014;12:290-6.

16 Sweetland S, Green J, Liu B, et al. Duration and magnitude of the postoperative risk of venous thromboembolism in middle aged women: prospective cohort study. BMJ 2009;339:b4583-.

17 Maestre A, Sánchez R, Rosa V, et al. Clinical characteristics and outcome of inpatients versus outpatients with venous thromboembolism: findings from the RIETE Registry. Eur J Intern Med 2010;21:377-82.

18 Medicare USA. Are you an inpatient or an outpatient? | Medicare. gov. https://www.medicare.gov/what-medicare-covers/part-a/ inpatient-or-outpatient.html (accessed 17 Aug 2017).

19 Heit JA, Crusan DJ, Ashrani AA, et al. Effect of a nearuniversal hospitalization-based prophylaxis regimen on annual number of venous thromboembolism events in the US. Blood 2017;130:109-14.

20 Bilimoria $\mathrm{KY}$, Chung J, Ju $\mathrm{MH}$, et al. Evaluation of surveillance bias and the validity of the venous thromboembolism quality measure. JAMA 2013;310:1482-9. 